ESAIM: PROCEEDINGS, November 2002, Vol.12, 115-128

M.Thiriet, Editor

\title{
SHORT-TERM MODELLING OF THE CONTROLLED CARDIOVASCULAR SYSTEM
}

\author{
A. Monti, C. MÉdigue, M. Sorine ${ }^{1}$
}

\begin{abstract}
Variables involved in cardiovascular regulation, such as blood flow and pressure, oxygen concentration, are under the control of several feedback mechanisms with different dynamics. We are interested in the short-term control of blood flow and pressure by the autonomic nervous system through baroreceptor control loops. The aim of this article is to relate usually measured cardiovascular signals to models of the controlled cardiovascular system. A multiple-feedback loop organisation is obtained that can help to study the control function. Control assessment could use signal-based estimates of associated feedback sensitivities.
\end{abstract}

Résumé. Les variables intervenant dans la régulation du système cardiovasculaire tels les débits, pressions, saturation du sang en oxygène, sont contrôlées par des mécanismes ayant différentes dynamiques. Nous sommes intéressés par le contrôle à court terme du débit et de la pression par le système nerveux autonome au moyen d'arcs baroréflexes. L'objectif de cet article est d'associer les signaux cardiovasculaires usuellement mesurés à des modèles du système cardiovasculaire et de son contrôle. La structure obtenue, à plusieurs boucles de feedback, peut aider à étudier la fonction de contrôle. Les sensibilités associées aux feedback, estimées à partir des signaux, pourraient servir à évaluer cette fonction.

\section{INTRODUCTION}

The function of the circulation is to supply tissues with oxygen, nutrients and to remove carbon dioxide and other catabolits. The organs involved in this function are: the lungs which allow gas exchanges, the heart which pumps blood and the vascular system which carries molecules to the tissues. Variables involved in cardiovascular regulation, such as blood flow, blood pressure level, oxygen blood concentration, are under the control of the autonomic nervous system (ANS). We are interested only on the short term control, about a few minutes, of the cardiovascular system in order to understand the functioning of the ANS: its physiological and pathological conditions. ANS controls the cardiovascular system by the sympathetic and parasympathetic neural innervation which have an antagonistic effect on the heart. Sympathetic nerve has a slow vasoconstrictor effect on the vessels and affects aortic and carotidien baroreceptors. Respiratory system has a central effect on cardiovascular control since the respiratory and cardiovascular centers, in the ANS, may interact each other. The respiratory activity has also a mechanical effect on vascular circulation by stretching and/or collapsing pulmonary and thoracic vessels. A good autonomic function is of crucial importance for life and is of great prognostic value in many diseases. The aim of this article is to relate cardiovascular signal analysis to models of the cardiovascular and control systems taking into account its multiple feedback loop organisation.

${ }^{1}$ INRIA Rocquencourt, France

(C) EDP Sciences, SMAI 2003 


\section{Cardiovascular system modelling}

The cardiovascular system consists of a complex double chamber pump (the heart), pumping a complex fluid (the blood) into complex pipes (the blood vessels) organised into vascular compartments, as shown in fig. (1). In fig. (1), the systemic vascular system is split into three main parts evidencing the measures available by ANS

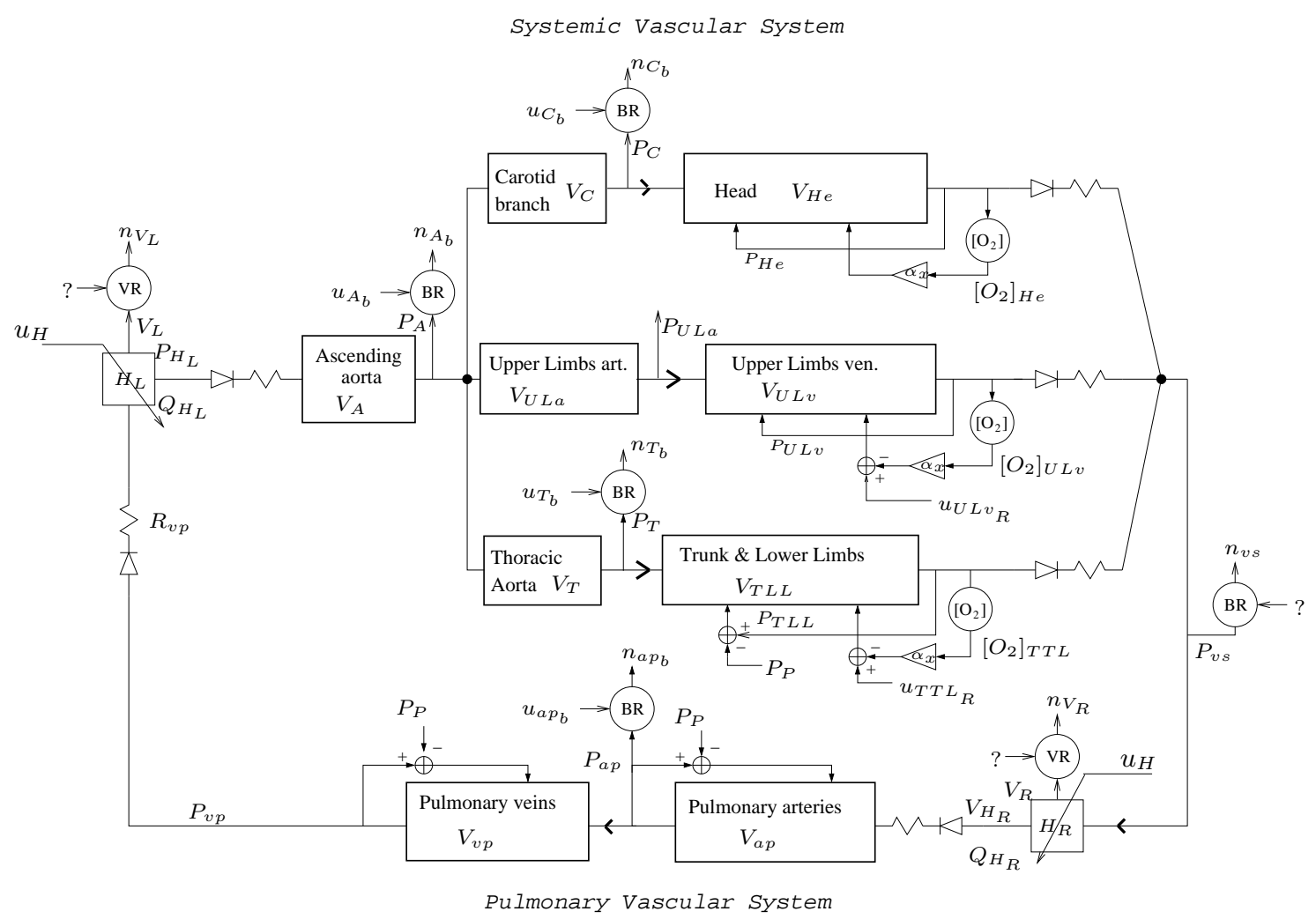

Figure 1. Diagram of the cardiovascular system. $H_{R}\left(H_{L}\right)$ is the right (left) part of the heart. $V_{R}\left(V_{L}\right)$ is the right (left) ventricle-volume, measured by voloreceptors (VR). We do not know if voloreceptors are controlled by the ANS. Vascular compartments are characterised by their volume/relative-pressure relationships $\mathcal{V}_{x}\left(\Delta P_{x}\right)$ and pressures $P_{x}$ or volumes $V_{x}(x=A$, $C$, He, ULa, ULv, T, TLL, ap, vp). The arterial pressure $P_{U L a}$ is measured at the upper limbs by external devices. $P_{x}(x=A, C, T$, ap, vs) are the measured blood pressure at different points in the cardiovascular system by ANS-controlled baroreceptors (BR). We do not know if the baroreceptor in the vs compartment is controlled by the ANS. This system is under control of the ANS (inputs $u_{H}, u_{U L v_{R}}, u_{T L L_{o}}, u_{A_{b}}, u_{C_{b}}, u_{T_{b}}, u_{a p_{b}}$ ), under respiratory mechanical influence (input $P_{P}$, pulmonary pressure) and under local control of blood oxygen concentration (input $\left.\left[\mathrm{O}_{2}\right]_{\mathrm{He}},\left[\mathrm{O}_{2}\right]_{U l v},\left[\mathrm{O}_{2}\right]_{T L L}\right)$. The diodes and the resistances stand for $a Q=|\Delta P|_{+} / R$ characteristic where $\Delta P$ is the blood pressure drop across the diode.

to control the cardiovascular system. These measures are provided by aortic $n_{A_{b}}$, carotid $n_{C_{b}}$, low-pressure arterial pulmonary $n_{a p_{b}}$, and thoracic aorta $n_{T_{b}}$ baroreceptors and by stretchreceptors localised in the ventricle walls $n_{V_{R}}$ and $n_{V_{L}}$. In addition, in classical clinical studies, a non invasive measure of arterial blood pressure is taken on the upper limb arteries by FINAPRES device or other instruments $P_{U L a}$.

In the following, we will develop the elements showed in fig.(1). 


\subsection{Passive vascular compartment modelling}

The vessels are grouped into a small number of vascular compartments (see fig.(1)). The relationship between lumped and distributed compartment-models is useful for interpreting the coefficients of reduced models. A distributed model of a compartment $x$ can be taken as a on one-dimensional flow equations [15]:

$$
\left\{\begin{array}{l}
\frac{\partial A_{x}}{\partial t}+\frac{\partial Q_{x}}{\partial z}=0, \quad c_{u} \frac{\partial Q_{x}}{\partial t}+\frac{\partial}{\partial z}\left(\frac{Q_{x}^{2}}{A_{x}}\right)=-\frac{A_{x}}{\rho} \frac{\partial P_{x}}{\partial z}-\frac{\lambda}{\rho} \frac{Q_{x}}{A_{x}} \\
P_{\mid z=0}=P_{i n_{x}}, \quad Q_{\mid z=L_{x}}=-\frac{1}{\lambda} \mathcal{A}_{x}^{2}\left(\Delta P_{x}\right) \frac{\partial P}{\partial z} \\
\mid z=L_{x}
\end{array}\right.
$$

$A_{x}$ is the equivalent cross-sectional area, $Q_{x}$ and $P_{x}$ the distributed blood flow and pressure, $c_{u}$ and $\lambda$ the coefficients of inertia and viscous terms, $\rho$ is the blood density. In the example of boundary conditions, $L_{x}$ is the compartment length. The system of equations is closed by a model of the local compliance of the compartment wall, $A_{x}=\mathcal{A}_{x}\left(\Delta P_{x}\right)$, with $\Delta P_{x}=P_{x}-P_{x_{o}}$ and $P_{x_{o}}$ the external pressure. For example, [15] proposes this simple 3 -parameters model:

$$
\mathcal{A}_{x}\left(\Delta P_{x}\right)=A_{x_{o}}\left[1+C_{x_{o}} \Delta P_{x}+C_{x_{1}} \Delta P_{x}^{2}\right]
$$

Windkessel models are obtained by neglecting acceleration terms in (1) which, with (2), becomes:

$$
\frac{\partial \mathcal{A}_{x}\left(P_{x}-P_{x_{o}}\right)}{\partial P_{x}} \frac{\partial P_{x}}{\partial t}-\frac{1}{\lambda} \frac{\partial}{\partial z}\left(\mathcal{A}_{x}^{2}\left(P_{x}-P_{x_{o}}\right) \frac{\partial P_{x}}{\partial z}\right)=0, \quad Q_{x}=-\frac{1}{\lambda} \mathcal{A}_{x}^{2}\left(P_{x}-P_{x_{o}}\right) \frac{\partial P_{x}}{\partial z}
$$

A crude approximation of (3) using only three points, leads to the reduced model where $P_{i n_{x}}, Q_{i n_{x}}$ and $P_{x}, Q_{x}$ are the pressure and flow at inlet and oulet respectively:

$$
L_{x} \frac{d \mathcal{A}_{x}\left(\Delta P_{x}\right)}{d \Delta P_{x}} \frac{d P_{x}}{d t}+Q_{x}+\frac{\mathcal{A}_{x}^{2}\left(\Delta P_{x}\right)}{\lambda L_{x}}\left(P_{x}-P_{i n_{x}}\right)=0, \quad Q_{i n_{x}}=-\frac{\mathcal{A}_{x}^{2}\left(\Delta P_{x}\right)}{\lambda L_{x}}\left(P_{x}-P_{i n_{x}}\right)
$$

The compartment lumped compliance and resistance define respectively, the blood-volume change due to a distending-pressure change and the blood-pressure drop due to an inlet flow. They are expressed as:

$$
C_{x}\left(\Delta P_{x}\right)=L_{x} \frac{d \mathcal{A}_{x}\left(\Delta P_{x}\right)}{d \Delta P_{x}}, \quad R_{x}\left(\Delta P_{x}\right)=\frac{\lambda L_{x}}{\mathcal{A}_{x}^{2}\left(\Delta P_{x}\right)}
$$

The usual description of the circulation leads to a clear separation between the resistance and compliance functions of the different compartments. We recover this property noting that in large arteries and veins, the resistance can be neglected and in arterioles, the blood volume changes can be neglected, the input pressure being filtered by upstream vascular compartiments.

Remark that classical 2-element Windkessel lumped model of the entire vascular compartiment $[14,17]$ can be obtained by two compartment models (4) in series by neglecting the aortic resistance in the first compartment and the peripheral systemic compliance in the second compartment. Similarly, the 3-element Windkessel model $[14,17]$ can be deduced by the series of two models (4) by taking into account the aorta resistance and neglecting the peripheral compliance. In addition, taking into account the acceleration term in (1), the corresponding reduced model would include the inertance term recently added to the 3 -WK model [14]. 
For control purposes it is convenient to represent the state of a compartment $x$ by its output pressure, $P_{x}$ or its volume $V_{x}$. We note $\mathcal{V}_{x}(\Delta P)=L_{x} \mathcal{A}_{x}(\Delta P)$, so that (4) finally leads to:

$$
\left\{\begin{array}{l}
\frac{d V_{x}}{d t}+Q_{x}+\frac{V_{x}^{2}}{\lambda L_{x}^{3}}\left(\mathcal{V}_{x}^{-1}\left(V_{x}\right)+P_{x_{o}}-P_{i n_{x}}\right)=0 \\
P_{x}=-\mathcal{V}_{x}^{-1}\left(V_{x}\right)+P_{x_{o}}, \quad Q_{i n_{x}}=-\frac{V_{x}^{2}}{\lambda L_{x}^{3}}\left(P_{x}-P_{i n_{x}}\right)
\end{array}\right.
$$

$P_{x_{o}}$ can be used as a perturbation input (e.g. pulmonary pressure for $x=v p, \ldots$ ) or as a controlled input for active compartments, as seen later.

\subsection{Heart Modelling}

A model of muscle contraction compatible with descriptions at different scales (molecular nanomotors, sarcomeres, myofibres) has been recently proposed [1,2]. It is used in distributed models of the heart, see [13]. Supposing a cylindrical ventricle-geometry, it leads to this model of (left for the example) ventricle, useful for studying the ANS control:

$$
\left\{\begin{array}{l}
\dot{k}_{c_{L}}=-\left(\left|u_{H}\right|+\left|\dot{\varepsilon}_{c_{L}}\right|\right) k_{c_{L}}+k_{c_{L o}}\left|u_{H}\right|_{+} \\
\dot{\sigma}_{c_{L}}=-\left(\left|u_{H}\right|+\left|\dot{\varepsilon}_{c_{L}}\right|\right) \sigma_{c_{L}}+k_{c_{L}} \dot{\varepsilon}_{c_{L}}+\sigma_{c_{L o}}\left|u_{H}\right|_{+} \\
\ddot{\varepsilon}_{c_{L}}=-(2 \zeta \omega+\eta \alpha) \dot{\varepsilon}_{c_{L}}-\omega^{2} \varepsilon_{c_{L}}-\alpha d_{o}\left(\varepsilon_{c_{L}}\right)\left(k_{c_{L}} \xi_{0}+\sigma_{c_{L}}\right)+\beta\left(\sqrt{V_{H_{L} / V_{H_{H}}}-1}\right) \\
\dot{V}_{H_{L}}=\frac{1}{R_{p v}}\left|P_{p v}-k_{L} \frac{d_{o}\left(\varepsilon_{c_{L}}\right)}{V_{H_{L}}} \sigma_{c_{L}}\right|_{+}-\frac{1}{R_{A}}\left|k_{L} \frac{d_{o}\left(\varepsilon_{c_{L}}\right)}{V_{H_{L}}} \sigma_{c_{L}}-P_{A}\right|_{+} \\
P_{H_{L}}=k_{L} \frac{d_{o}\left(\varepsilon_{c_{L}}\right)}{V_{H_{L}}} \sigma_{c_{L}}
\end{array}\right.
$$

where

- $k_{c_{L}}$ and $\sigma_{c_{L}}$ are the total left ventricle muscular controlled stiffness and stress;

- $\varepsilon_{c_{L}}$ is the left ventricle wall strain;

- $d_{o}\left(\varepsilon_{c_{L}}\right)$ is the lenght-tension relationship of the muscle fibers;

- $P_{H_{L}}$ and $V_{H_{L}}$ are the left ventricle pressure and volume;

- $P_{p v}, P_{A}$ and $R_{p v}$ are shown in fig.(1) and $R_{A}$ is the blood-flow resistance in the aortic compartment;

- $u_{H}(t)$ is the electro-chemical command controlling the left ventricle contraction.

$d_{o}(\varepsilon)$ appears as a mechanical gain adaptation which takes into account the Starling effect, see fig.(2): in the

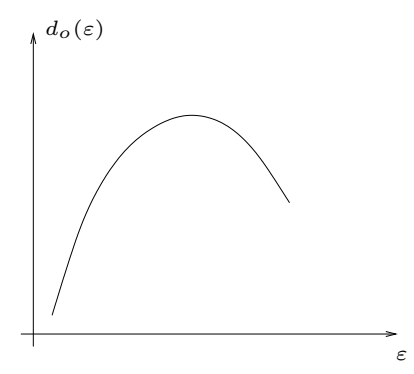

FiguRE 2. Mechanical command $d_{o}(\varepsilon)$ of the heart to take into account the Starling effect.

ascending limb of the Starling curve, the more is filled the heart the more amount of blood the pump will push out; in the descending limb the more is filled the heart the smaller amount of blood the pump will push out. Assuming a separation of the mechanical and chemical command, we are lead to conceive a hierarchical control 
of the heart. At the upper level $u_{H}(t)$ is the command which is applied to guarantee a good oxygenation of the body tissue assuming a steady filling of the heart, i.e. the controller acts as the heart pumps in a open loop system. At the lowest level, given a central command $u_{H}(t)$ and the actual filling condition of the heart, $d_{o}\left(\varepsilon_{c_{L}}\right)$ regulates the command $u_{H}(t)$ to finally have the correct heart command. Remark that, in the ventricular and atrium walls are located stretchreceptors that can be used by the ANS to estimate this $d_{o}\left(\varepsilon_{c_{L}}\right)$ regulation.

Other models based on a time variant elastance [5] have been proposed, but, being behavioural models, their parameters and inputs have less direct physiological meaning.

\subsection{Baroreceptor Modelling}

Physiology. When a change in carotid sinus (or in the aorta) arterial pressure occurs, the viscoelastic wall, where the receptors at the nerve endings are located, is deformed. The mechano-electrical transduction takes place in these receptors by a poorly understood coupling mechanism: pulses are sent on the baroreceptor nerves and their firing rate, the baroreceptor activity, is changed in response to pressure change. The main characteristics of carotid sinus baroreceptor response exhibit complex dynamics and nonlinearities:

- firing rate increases with carotid pressure;

- response curves exhibit threshold (the positive and stable equilibrium value which the firing rate approaches when no change in pressure appears) and saturation (maximum value of the firing rate) in a sigmoidal shape and show asymmetric behaviour depending on the direction of pressure changes. The observed sigmoidal pressure-firing rate relationship seems to be more related to the deformation-firing rate transduction process than to the pressure-deformation transduction process;

- sufficiently fast decreases in pressure cause firing to drop even below the threshold value;

- a step change in pressure causes a step change in firing rate followed by resetting phenomenon, i.e. a decay in firing rate towards the threshold value. Resetting is called adaptation;

- there are smooth muscles located within the adventitial smooth muscles of vessel wall which are innervated by sympathetic efferents. Stimulation of these efferents causes an increase in the baroreceptor nerve activity, a decrease in the carotid sinus diameter and its elastic modulus. This effect is evident for intrasinus pressure below $130 \mathrm{mmHg}$, above $130 \mathrm{mmHg}$ this effect is largely reduced and it disappears at very high intrasinus pressures;

- finally, observations indicate that the response curves for hypotensive sinus and hypertensive sinus simply are translations to the left and right along the pressure axis, respectively, of the response curve for normotensive sinus.

More details and baroreceptors models can be found in $[7,11,16]$. But none of these models separate the pressure-deformation of the carotid sinus wall model from the mechano-electrical transduction in the receptors. In fact, they describe responses to stress changes rather than to stress: they cannot be used as pressure-sensor models. Moreover, none of them take into account the efferent sympathetic influence on baroreceptor activity, as discussed below, even if in [7], a first step in this direction has been made. In [10] we have proposed a simple algorithm that the SNA could use to estimate pressure from the baroreceptor activity by exploiting the presence of a threshold in the pressure-deformation relation. This suggests the possible usefulness of the sympathetic feedback for baroreceptor activity control.

Modelling of the pressure-deformation transduction process. The vessel wall is constituted by both smooth muscles, collagens and elastic membrane constituting three coats: intima, media and adventitia. Thick adventitia layer consists of a network of collagenous fibers within which smooth muscle cells are interspersed in a parallel organisation and which encases the external elastic lamina. The special arrangement of collagen and smooth muscle within the arterial wall forms a "jacket" which controls the distensibility of the artery. The stretch-receptors are in the middle of the vessel wall, in fact the baroreceptor nerve terminals are located along the media-adventitial border and in the deep layers of the adventitia of the sinus wall [7]. Similarly to [7], we can assume that baroreceptor activity originates from: (i) collagen receptors located within the adventitial collagenous fibers, $n_{\text {coll }}$ in fig.(3) (ii) elastin receptors located within the external elastic membrane, $n_{e}$ in 
fig.(3). In [10] it is shown that information provided by these receptors is sufficient for blood pressure transduction process. Smooth muscle receptors are not strictly necessary, therefore, they can provide complementary information or they does not exist. Clear information about this topic is not available.

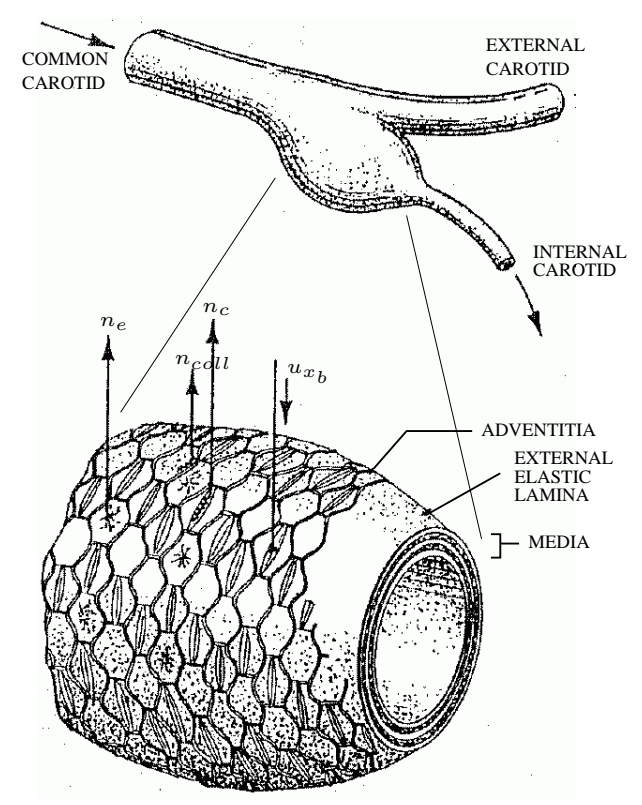

FIGURE 3. Spatial arrangement of baroreceptor (bottom) within the carotid sinus wall and their innervations, from [6, 7]. Baroreceptor activity originates from receptors linked to the three specific wall structures [7]: elastin, collagen fibers and smooth muscles (receptors outputs are respectively $\left.n_{e}, n_{\text {coll }}, n_{c}\right)$. The ANS controls smooth muscle contraction by local sympathetic efferent activity (control inputs: $u_{x_{b}}$ with $x=A, C, T$, ap).

Elastin element and Collagen fiber models [3]. A linear model links elastin fibers strain $\varepsilon_{e}$ and stress $\sigma_{e}$ and an idealized stress-strain model is considered for Collagen fibers, see fig.(4):

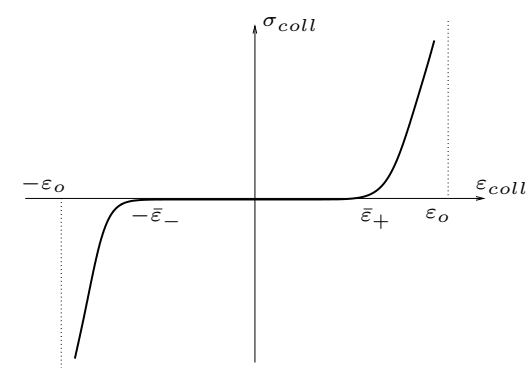

FIGURE 4. Idealized $\sigma_{\text {coll }}=\mathcal{H}_{\text {coll }}\left(\varepsilon_{\text {coll }}\right)$ relationship of collagen fibers.

$$
\sigma_{e}=k_{e} \varepsilon_{e}, \quad \sigma_{\text {coll }_{i}}=\mathcal{H}_{\text {coll }_{i}}\left(\varepsilon_{\text {coll }_{i}}\right)=k_{\text {coll }_{i}}\left|e^{\frac{\varepsilon_{\text {coll }_{i}}-\varepsilon_{i+}}{\tau_{i+}}}-1\right|_{+}, \quad i=1,2 \quad \text { for } \varepsilon_{\text {coll }_{i}} \ll \varepsilon_{o}
$$

Smooth muscle model. This model, $\sigma_{x_{b}}=\mathcal{H}_{x_{b}}\left(\varepsilon_{x}, u_{x_{b}}\right)$, for baroreceptors $(x=A, C, T$, ap, vs, see fig.(1)) is a version without active relaxation $\left(u_{x_{b}} \geq 0\right)$ of the contractile-element model of $[1,2]$, already introduced in 
paragraph 1.2 for heart modelling. For a contractile element $c$, this model is:

$$
\left\{\begin{array}{l}
\dot{k}_{c}=-\left(u_{x_{b}}+\left|\dot{\varepsilon}_{c}\right|\right) k_{c}+k_{c_{0}} u_{x_{b}} \\
\dot{\tilde{\sigma}}_{c}=-\left(u_{x_{b}}+\left|\dot{\varepsilon}_{c}\right|\right) \tilde{\sigma}_{c}+k_{c} \dot{\varepsilon}_{c}+\sigma_{c_{0}} u_{x_{b}} \\
\sigma_{c}=d_{o}\left(\varepsilon_{c}\right)\left(k_{c} \xi_{0}+\tilde{\sigma}_{c}\right)+\eta \dot{\varepsilon}_{c}
\end{array}\right.
$$

Vessel wall model. The organisation of elastin, collagen and contractile elements of fig.(5) implies, for small deformations and wall thickness, the following relations where the parameters are constant lengths and the elastin stiffness: $\sigma_{\text {coll }_{1}} \bar{h}_{1}=\sigma_{c} \bar{h}_{c}+\sigma_{\text {coll }_{2}} \bar{h}_{\text {coll }_{2}}$ and, for the inner baroreceptor pressure $P_{x_{b}}$ :

$$
P_{x_{b}}=\frac{2 \pi\left(\sigma_{\text {coll }_{1}} \bar{h}_{1}+\sigma_{e} \bar{h}_{e}\right)}{\bar{l}_{e}\left(1+\sigma_{e} / k_{e}\right)}+P_{x_{0}}
$$

So the ANS can estimate $P_{x_{b}}-P_{x_{0}}$ from the knowledge of collagen and elastin stresses, $\sigma_{\text {coll }}$ and $\sigma_{e}$.

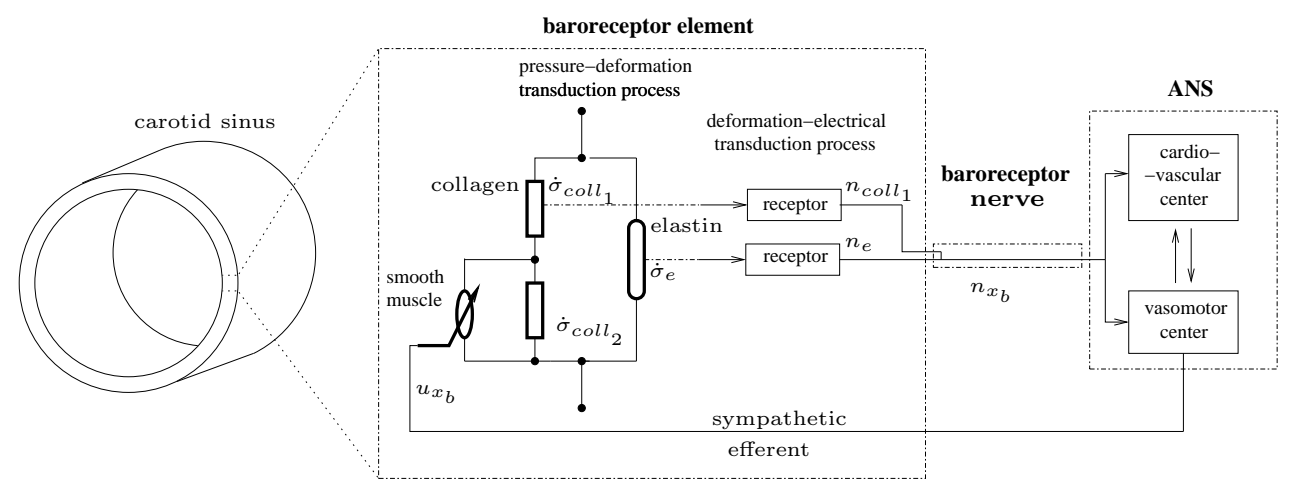

FIGURE 5. Model of spatial arrangement of elastin, collagen and smooth muscle elements within the carotid sinus wall, see fig.(3). $u_{x_{b}}$ is the sympathetic control of the slow and weak smooth muscles and $n_{x_{b}}$ is the firing rate recorded on the baroreceptor nerve.

Model of the deformation-firing rate transduction process. We use a simplified version of the model proposed by Ottesen in [11] which relates intrasinus pressure to neural activity: only two firing rate ranges are considered here to code $\dot{\sigma}_{\text {coll }_{1}}$ and $\dot{\sigma}_{e}$. Remark that stretchreceptors are sensible to the time derivative of the stresses:

$$
\left\{\begin{array}{l}
\dot{n}_{\text {coll }_{1}}=\kappa_{\text {coll }_{1}} \dot{\sigma}_{\text {coll }_{1}} \frac{n_{\text {coll }_{1}}\left(M_{\text {coll }_{1}}-n_{\text {coll }_{1}}\right)}{\left(M_{\text {coll } \left._{1} / 2\right)^{2}}\right.}-\frac{1}{\tau_{\text {coll }_{1}}}\left(n_{\text {coll }_{1}}-N_{\text {coll }_{1}}\right) \\
\dot{n}_{e}=\kappa_{e} \dot{\sigma}_{e} \frac{n_{e}\left(M_{e}-n_{e}\right)}{\left(M_{e} / 2\right)^{2}}-\frac{1}{\tau_{e}}\left(n_{e}-N_{e}\right), \quad x=A, C, T, \text { ap } \\
n_{x_{b}}=\kappa_{1} n_{\text {coll }_{1}}+\kappa_{2} n_{e}
\end{array}\right.
$$

where the $\dot{n}$ 's denote the time derivatives of the firing rates $n$, the $\kappa$ 's are constants, $M$ the maximal firing rates, $\tau$ the characteristic time constants describing the resetting and $N$ the thresholds, i.e. the positive and stable equilibrium values which the firing rates approache when no change in pressure appears. Remark that (11) has, implicitly, all the cited characteristics of the baroreceptor response to blood pressure stimulation: asymmetric sigmoidal shape depending on the direction of strain changes, resetting mechanisms and, finally, it does not depend of strain value, therefore, it can be easily applied to normo-, hyper- or hypo-tensive subjects after a transient due to the baroreceptor adaptation. Ottesen, using (11), was able to reproduce classical experimental 
findings concerning the blood pressure-baroreceptor nerve activity relationship. A question arises: if (11) really describes the deformation-firing rate transduction process, how is it possible for the ANS to estimate the baroreceptor inner relative-pressure, $P_{x_{b}}-P_{x_{0}}$ ?

Pressure estimation by a tonic control of the weak, slow smooth muscle elements. The difficulty of estimation comes from the absence of a reference stress. We show that such a reference is available each time the stress goes out of the deadzone of the $\sigma-\varepsilon$ collagen characteristic: for these reference-instants, the value of $\sigma_{\text {coll }_{1}}$ and $\sigma_{e}$ will be known. This can occur maximum once each beat, if the deadzone boundary is in the strain range which can be a natural property or obtained by a tonic control of the weak, slow smooth muscle elements. The reference-instants correspond to events easy to detect in the history of $\dot{\sigma}_{\text {coll }_{1}}$ (changes of the mean value). The tonic control of smooth muscle can simply be $u_{x_{b}}=0$, for high mean pressure values, and $u_{x_{b}}=u_{x}^{\text {max }}>0$ for low mean pressures values.

Estimation problem: Estimate collagen and elastin stresses $\sigma_{\text {coll }}(t), \sigma_{e}(t)$ (and hence $P_{x_{b}}-P_{\text {ext }}$ ) from their $\overline{\text { time derivatives } \dot{\sigma}_{\text {coll }}}(t), \dot{\sigma}_{e}(t)$.

Assumptions

- an alternance of diastolic $\left(\dot{P}_{x_{b}} \leq 0\right)$ and systolic $\left(\dot{P}_{x_{b}}>0\right)$ phases takes place. We note $T_{n}=\left[t_{n}, t_{n+1}\right]$ so that $T_{2 n}$ will represent diastolic phases and $T_{2 n+1}$ systolic phases, $n=0,1 \ldots$

- collagen characteristic is idealized, as shown in fig.(4), and suppose that ANS has an a priori estimation of $k_{e}$ and of $\bar{\varepsilon}_{i}(i=1,2)$, the upper limits of the deadzone;

- $\sigma_{c}$ is nearly constant, in the following we suppose $\sigma_{c}=\bar{\sigma}_{c} \geq 0$. Then, $\dot{\sigma}_{\text {coll }_{1}}=\frac{\bar{h}_{\text {coll }_{2}}}{h_{c}+h_{\text {coll }_{2}}} \dot{\sigma}_{\text {coll }_{2}}$;

- suppose there is always an instant in the recent past where collagen entered into the deadzone. More precisely, noting $z(n)=\max \left\{n_{o} \mid n_{o} \leq n, \dot{\sigma}_{\text {coll }_{1}}\left(t_{2 n_{o}}\right)<0\right.$ and $\left.\exists t \in T_{2 n_{o}}, \dot{\sigma}_{\text {coll }_{1}}(t)=0\right\}$ we assume that $\sup _{n}(n-z(n))<+\infty$. In the following, we will note:

$$
\bar{t}_{2 z(n)}=\min \left\{t \mid t \in T_{2 z(n)}, \dot{\sigma}_{\text {coll }_{1}}(t)=0\right\}
$$

$\underline{\text { Properties }[10]: ~}$

$$
\forall t \dot{\sigma}_{e}(t) \neq 0 \text { and } \dot{\sigma}_{\text {coll }_{i}}(t)=0(i=1,2) \Rightarrow \sigma_{\text {coll }_{1}}(t) \sigma_{\text {coll }_{2}}(t)=0
$$

$$
\begin{aligned}
& \forall n, \forall t \in T_{2 n} \dot{P}_{x_{b}}(t) \leq 0 \Rightarrow \dot{\sigma}_{e}(t) \leq 0 \text { and } \dot{\sigma}_{\text {coll }}(t) \leq 0 \\
& \forall n, \forall t \in T_{2 n+1} \dot{P}_{x_{b}}(t)>0 \Rightarrow \dot{\sigma}_{e}(t)>0 \text { and } \dot{\sigma}_{\text {coll }}(t) \geq 0
\end{aligned}
$$

Estimation of $P_{x_{b}}(t)-P_{x_{0}}[10] . \bar{t}_{2 z(n)}$ being given by (12), we have:

$$
\left\{\begin{array}{l}
\hat{\sigma}_{\text {coll }_{1}}(t)=\hat{\sigma}_{\text {coll }_{1}}\left(\bar{t}_{2 z(n)}\right)+\int_{\bar{t}_{2 z(n)}}^{t} \dot{\sigma}_{\text {coll }_{1}}(t) d t \\
\hat{\sigma}_{e}(t)=\hat{\sigma}_{e}\left(\bar{t}_{2 z(n)}\right)+\int_{\bar{t}_{2 z(n)}}^{t} \dot{\sigma}_{e}(t) d t \\
\hat{P}_{x_{b}}(t)-\hat{P}_{x_{0}}=\frac{2 \pi\left(\hat{\sigma}_{\text {coll }_{1}}(t) \bar{h}_{\text {coll }_{1}}+\hat{\sigma}_{e}(t) \bar{h}_{e}\right)}{\bar{l}_{e}\left(1+\hat{\sigma}_{e}(t) / k_{e}\right)}
\end{array}\right.
$$

where

$$
\left\{\begin{array}{l}
\hat{\sigma}_{e}\left(\bar{t}_{2 z(n)}\right)=k_{e}\left(\frac{\bar{l}_{\text {coll }_{1}}\left(1+\max \left(\varepsilon_{1+}, \mathcal{H}_{\text {coll }_{1}}^{-1}\left(\bar{\sigma}_{c}\right)\right)+\bar{l}_{\text {coll }_{2}}\left(1+\varepsilon_{2+}\right)\right.}{\bar{l}_{e}}-1\right) \\
\hat{\sigma}_{\text {coll }_{1}}\left(\bar{t}_{2 z(n)}\right)=\bar{\sigma}_{c} \frac{\bar{h}_{c}}{\bar{h}_{c}+\bar{h}_{\text {coll }_{2}}}
\end{array}\right.
$$

\section{Comments:}

(1) The estimation of $P_{x_{b}}(t)-P_{e x t}$ is based on the detection of "buckled collagen fiber" condition during a local diastolic phase; 
(2) The oscillating behaviour of the inside blood pressure allows intrasinus blood pressure estimation, in steady condition blood pressure estimation will be not possible.

(3) The presence of both elastin elements and collagen fibers is needed for blood pressure estimation.

(4) Blood pressure estimation is finally very simple and needs the knowledge of $k_{e}, \bar{\varepsilon}_{i+}(i=1,2), \bar{\sigma}_{c}$ and an accurate observation of the sign of $\dot{\sigma}_{\text {coll }}$ and the history of $\dot{\sigma}_{\text {coll }}$ and $\dot{\sigma}_{e}$.

(5) Blood pressure estimation can be made by a very simple smooth muscle control law: $\bar{\sigma}_{c}=0$ or $\bar{\sigma}_{c}=$ $\sigma_{c}^{\max }>0$. By (13) we can conclude that active blood pressure estimation $\left(\bar{\sigma}_{c}=\sigma_{c}^{\max }\right)$ is useful only for high blood pressure values, whereas passive blood pressure estimation without smooth muscle contraction $\left(\bar{\sigma}_{c}=0\right)$ can be used for low blood pressure values, using (14).

In the following, we assume that the ANS knows the blood pressures $P_{x_{b}}-P_{x_{0}}$ at baroreceptor positions.

\subsection{Active vascular compartment modelling}

We are now interested to model an active vascular compartment, as the one shown in fig.(6). The aim is to find a dynamical relation between the volume $V_{x}$, the pressure difference $P_{x}-P_{x_{o}}$ and the command $u_{x}$ of smooth muscles of the vascular compartment $x$, with $x=U L v, T T L$, see fig.(1). We assume that the vascular

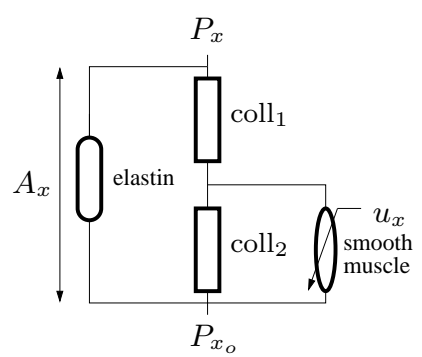

FiguRE 6. Simplified rheological model of a vascular compartment $x . u_{x}$ is the command of smooth muscles. $P_{x_{o}}$ is an external pressure that can be considered as a constant perturbation. $P_{x}$ is the blood pressure in the vascular compartment. $A_{x}$ is the cross-sectional area of the vascular compartment. Assuming a constant length of the vascular compartment $\bar{l}_{x}$, the volume of the $x$ vascular compartment is given by $V_{x}=\bar{l}_{x} A_{x}$.

wall structure is similar to baroreceptor wall structure, so the rheological diagram shown in fig.(6) is the same as the one shown in fig.(5). To describe collagen, elastin and smooth muscle elements, we consider the same stress-strain relationships introduced at paragraph 1.3.

Considering eqs. (8), (9), (10), we can write:

$$
\left\{\begin{array}{l}
\varepsilon_{e}\left(V_{x}\right)=\sqrt{\frac{4 \pi V_{x}}{\bar{l}_{e}^{2} \bar{l}_{x}}}-1 \\
\varepsilon_{\text {coll }_{2}}\left(\varepsilon_{c_{x}}\right)=\frac{\bar{l}_{c}}{\bar{l}_{\text {coll }_{2}}}\left(1+\varepsilon_{c_{x}}\right)-1 \\
\varepsilon_{\text {coll }_{1}}\left(V_{x}, \varepsilon_{c_{x}}\right)=\frac{\bar{l}_{e}}{\bar{l}_{\text {coll }_{1}}}\left(1+\varepsilon_{e}\left(V_{x}\right)\right)-\frac{\bar{l}_{c}}{\bar{l}_{\text {coll }_{1}}}\left(1+\varepsilon_{c_{x}}\right)-1 \\
\sigma_{c_{x}}\left(V_{x}, \varepsilon_{c_{x}}\right)=\frac{\bar{h}_{\text {coll }_{2}}}{h_{c}} \mathcal{H}_{\text {coll }_{2}}\left(\varepsilon_{\text {coll }_{2}}\left(\varepsilon_{c_{x}}\right)\right)
\end{array}\right.
$$


Finally, a dynamic model of the active Windkessel compartment has four state variables: $k_{c_{x}}, \tilde{\sigma}_{c_{x}}, \varepsilon_{c_{x}}, V_{x}$. Considering $P_{i n}$ and $Q_{x}$ as inputs of the model (6), the active compartment model is:

$$
\begin{cases}\dot{k}_{c_{x}} & =-\left(u_{x}+\left|\dot{\varepsilon}_{c_{x}}\right|\right) k_{c_{x}}+k_{c_{x_{o}}} u_{x} \\ \dot{\tilde{\sigma}}_{c_{x}} & =-\left(u_{x}+\left|\dot{\varepsilon}_{c_{x}}\right|\right) \tilde{\sigma}_{c_{x}}+k_{c_{x}} \dot{\varepsilon}_{c_{x}}+\sigma_{c_{x_{o}}} u_{x} \\ \dot{\varepsilon}_{c_{x}} & =\frac{1}{\eta_{x}}\left(d_{o}\left(\varepsilon_{c_{x}}\right)\left(k_{c_{x}} \xi_{0}+\tilde{\sigma}_{c_{x}}\right)-\sigma_{c_{x}}\left(V_{x}, \varepsilon_{c_{x}}\right)\right) \\ \dot{V}_{x} & =-Q_{x}-\frac{V_{x}^{2}}{\lambda \bar{l}_{x}^{3}}\left(\Delta P_{x}\left(V_{x}, \varepsilon_{c_{x}}\right)-P_{i n}+P_{x_{o}}\right) \\ \text { where } & \Delta P_{x}\left(V_{x}, \varepsilon_{c_{x}}\right)=2 \pi \frac{\bar{h}_{\text {coll }_{1}} \mathcal{H}_{\text {coll }_{1}}\left(\varepsilon_{c_{\text {oll }}}\left(V_{x}, \varepsilon_{c_{x}}\right)\right)+k_{e} \bar{h}_{e} \varepsilon_{e}\left(V_{x}\right)}{\bar{l}_{e}\left(1+\varepsilon_{e}\left(V_{x}\right)\right)}\end{cases}
$$

if $Q_{i n_{x}}$ is given instead of $P_{i n_{x}}$, we can use $Q_{i n}=\frac{V_{x}^{2}}{\lambda l_{x}^{3}}\left(\Delta P_{x}\left(V_{x}, \varepsilon_{c_{x}}\right)-P_{i n}+P_{x_{o}}\right)$.

Remark the similitude between the heart model (7) and this active vascular compartment model (15).

\section{Cardiovascular short-term Control modelling}

\subsection{Local control of vascular smooth muscles}

There is a local control of cross-sectional area-pressure relation as is well known in physiology [4]. We expressed it as a feedback law, see fig. (7):

$$
u_{x}=u_{x_{R}}-\alpha_{x}\left[O_{2}\right]_{x} \quad x=U L v, T L L
$$

The reference value, $u_{x_{R}}$, for smooth muscle command in the vascular compartment $x$ is provided by the $\alpha$

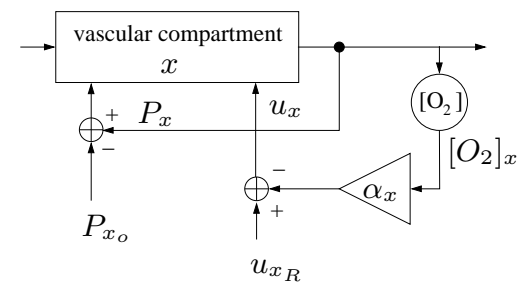

FIGURE 7. Functional diagram of the interaction between local and central control of vascular smooth muscle in a vascular compartment $x$.

sympathetic efferent nerves of the ANS. Locally, this set-point can be corrected or not to get a good supply of oxygen to working muscles.

\subsection{Beat-to-beat estimation of aortic blood flow}

We relate the time-average of the aortic blood-flow $Q_{A}$ to discrete-time cardiovascular variables showing a possible discrete-time feedback loop for the control of aortic blood flow compatible with the discrete-time nature of pressure measurements. Using results of paragraphs 1.1, 1.2, we have:

$$
\left\{\begin{array}{l}
\dot{V}_{L}=\frac{\left|P_{v p}-P_{H_{L}}\right|_{+}}{R_{v p}}-\frac{\left|P_{H_{L}}-P_{A}\right|_{+}}{R_{A}\left(P_{A}-P_{A_{o}}\right)} \\
Q_{A}=-C_{A}\left(P_{A}-P_{A_{o}}\right) \dot{P}_{A}+\left|\dot{V}_{L}\right|_{-}
\end{array}\right.
$$

We use here the definitions of compliance and resistance. For example, $C_{A}\left(\Delta P_{A}\right)=C_{A_{o}}+C_{A_{1}} \Delta P_{A}$. Assumptions: 


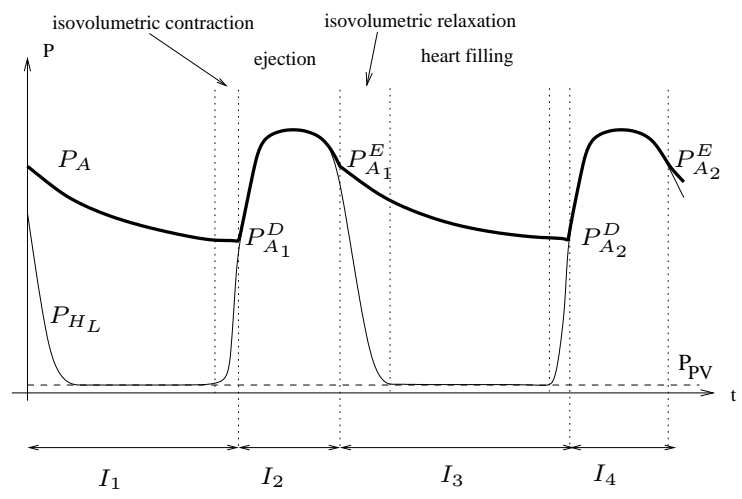

Figure 8. Idealised pressure curves in the left ventricle $P_{H_{L}}$ and in the ascending aorta $P_{A}$. The cardiac cycle is split into systolic and diastolic phases.

- an alternation of blood ejection $\left(\dot{V}_{L}<0\right)$ and heart relaxation, filling and iso-volumic contraction $\left(\dot{V}_{L} \geq 0\right)$ phases takes place on intervals $I_{m}=\left[t_{m}, t_{m+1}\right](m=0,1, \ldots): I_{2 m}$ represents the $m$-th heart ejection, $I_{2 m+1}$ represents the $m$-th heart relaxation, filling and iso-volumic contraction phase;

- we assume, and physiologicaly have, that $\forall t, P_{v p}(t)<P_{A}(t)$, i.e. blood pressure in the pulmonary venous compartment is always smaller than the pressure in the ascending aorta compartment;

\section{Notations:}

- $H R_{m}=1 /\left(t_{2 m}-t_{2 m-2}\right), m$-th instantaneous heart rate;

- $P_{A_{m}}^{D}=P_{A}\left(t_{2 m}\right), m$-th aortic diastolic pressure;

- $P_{A_{m}}^{E}=P_{A}\left(t_{2 m+1}\right)$, aortic pressure at the end of the $m$-th heart ejection;

- $V_{A_{m}}^{D}=V_{A}\left(t_{2 m}\right)$, blood volume in the ascending aorta compartment at the end of the $m$-th isovolumetric phase;

- $V_{A_{m}}^{E}=V_{A}\left(t_{2 m+1}\right)$, blood volume in the ascending aorta compartment at the end of the $m$-th ejection;

- $V_{L_{m}}^{D}=V_{L}\left(t_{2 m}\right)$, left ventricular volume at the end of the $m$-th isovolumetric phase;

- $V_{L_{m}}^{E}=V_{L}\left(t_{2 m+1}\right)$, left ventricular volume at the end of the $m$-th ejection.

Then, $\bar{Q}_{A_{m}} \stackrel{\text { def }}{=} H R_{m} \int_{t_{2 m-2}}^{t_{2 m}} Q_{A}(t) d t$ is given by (easy to prove):

$$
\bar{Q}_{A_{m}}=H R_{m}\left\{\left[C_{A}\left(P_{A_{m-1}}^{D}-P_{A_{o}}\right) P_{A_{m-1}}^{D}-C_{A}\left(P_{A_{m}}^{D}-P_{A_{o}}\right) P_{A_{m}}^{D}\right]+V_{L_{m-1}}^{D}-V_{L_{m-1}}^{E}\right\}
$$

\subsection{Feedbacks in cardiovascular short-term control}

ANS regulates blood flow and arterial blood pressure in a multi-input multi-output control loop organisation, see fig.(9). Sympathetic $u_{s}$ and parasympathetic $u_{v}$ have an antagonistic effect on the heart by $u_{H}$ input. Sympathetic component $u_{s}$ has a slow vasoconstrictor effect on the vessels by $u_{x_{R}}$ input where $x$ stands for a general active vascular compartment. In addition, $u_{s}$ affects baroreceptors by $u_{A_{b}}, u_{C_{b}}, u_{T_{b}}$ and $u_{a p_{b}}$ where $A_{b}$ stands for aortic, $C_{b}$ carotidien, $T_{b}$ thoracic aorta and $a p_{b}$ low pressure baroreceptors. Respiratory activity has a mechanical effect on vascular circulation by pulmonary pressure $P_{P}$ which stretches and collapses pulmonary and thoracic vessels. We can describe the control of the cardiovascular system in the standard form of a physical feedback system, as illustrated in fig.(9). The aortic, carotid, thoracic aorta, low pressure and vena cava baroreceptor are the pressure sensors which generate the afferent neural information $n_{A_{b}}, n_{C_{b}}, n_{T_{b}}, n_{a p_{b}}$ and $n_{v s}$. Ventricula voloreceptors are the volume receptors which generate $n_{V_{L}}$ and $n_{V_{R}}$. We can assume that ANS can estimate the aortic $\left(P_{A}\right)$, carotidian $\left(P_{C}\right)$, thoracic aorta $P_{T}$ and pulmonary blood pressure $P_{a p}$ as shown in the paragraph 1.3. In our point of view, the ANS may regulate blood flow in the different parts of 


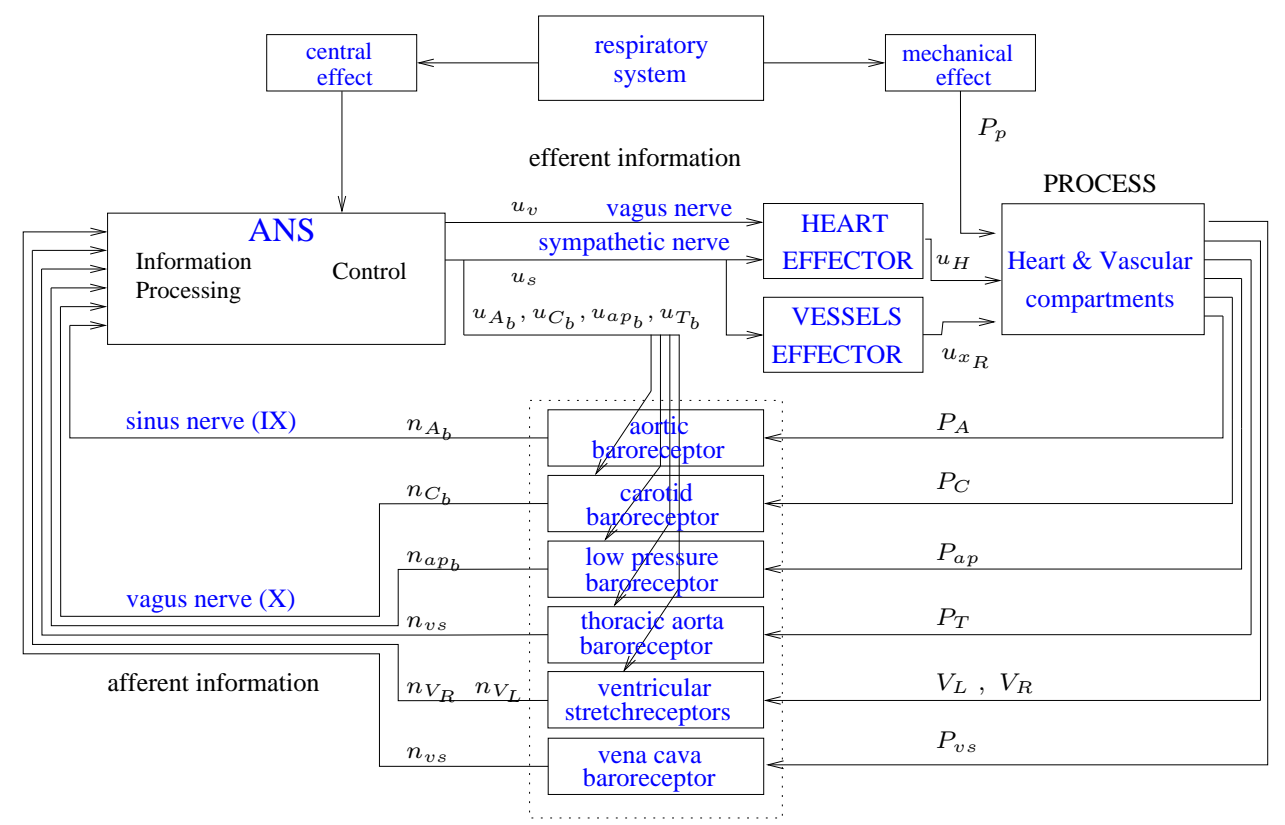

\section{FEEDBACK SENSORS}

FIGURE 9. Diagram of the feedback control loop in the cardiovascular system. ANS controls this system by the sympathetic and parasympathetic neural innervation, $u_{s}$ and $u_{v} . u_{H}$ is the heart contraction command. $u_{x_{R}}$ is the blood-vessel-constriction command, where $x$ stands for a generic vascular compartment. $u_{s}$ affects baroreceptors by $u_{A_{b}}, u_{C_{b}}, u_{T_{b}}$ and $u_{a p_{b}}$ where $A_{b}$ stands for aortic, $C_{b}$ carotidien, $T_{b}$ thoracic aorta and ap $p_{b}$ low pressure baroreceptors. Respiratory system has both a central and mechanical effect on cardiovascular control. Mechanical effect is function of the pulmonary pressure $P_{p} . P_{A}, P_{C}, P_{a p}, P_{T}, P_{v s}$ are the aortic, carotidian, pulmonary, thoracic aortic and vena cava blood-pressures. $V_{L}$ and $V_{R}$ are the left and right ventricular volumes.

the cardiovascular system, in the head as well as in the general systemic part, maintaining a particular sensible blood pressure, i.e. in the head, the lungs and the ascending aorta, within normal limits $\left(P_{x_{\min }} \leq P_{x} \leq P_{x_{\max }}\right.$, $\mathrm{x}=\mathrm{A}, \mathrm{C}, \mathrm{P})$ to avoid tissue damages and vessel collapses. In fact, blood flow could be estimated by afferent information, see paragraph 2.2, and the ANS could control effectors in order to maintain small the difference between the estimated blood flow and the reference value.

The study of control laws is under current research. A proportional control law, for example, could model the mechano-chemical heart control in a distributed structure:

$$
\left\{\begin{array}{l}
u_{H, n+1}=-\alpha_{A} e_{Q_{A_{n}}}-\sum_{x=A, P} \beta_{x}\left|P_{x}^{S}-P_{x_{\max }}\right|_{+}-\sum_{x=A, P} \gamma_{x}\left|P_{x_{\min }}-P_{x}^{S}\right|_{+} \\
u_{U L V_{R, n+1}}=-\frac{\alpha_{C}}{R_{C}}\left(\Delta P_{C}-\Delta P_{C_{R}}\right)+\beta_{C}\left|P_{C}^{S}-P_{C_{m a x}}\right|_{+}-\gamma_{C}\left|P_{C_{m i n}}-P_{C}^{S}\right|_{+} \\
u_{T L L_{R, n+1}}=-\frac{\alpha_{T}}{R_{T}}\left(\Delta P_{T}-\Delta P_{T_{R}}\right)+\beta_{T}\left|P_{T}^{S}-P_{T_{\max }}\right|_{+}-\gamma_{T}\left|P_{T_{\min }}-P_{T}^{S}\right|_{+}
\end{array}\right.
$$


where $\Delta P_{x}=P_{A}-P_{x}$. The command $u_{n+1}$ could control the cardiac function regulating the aortic blood flow and to maintain pressure in the aorta and in the lungs within normal limits. Remark that the ANS by the knowledge of $P_{A}(t)$ and $V_{L}(t)$ can estimate the error of blood flow regulation $e_{Q_{A_{n}}}$ in a beat-to-beat basis, cf. (18). Blood flow in the different blood compartments could be regulated by $u_{U L V_{n+1}}$ and $u_{T L L_{n+1}}$, which in fact control the pressure difference in the vascular system and, obviously, this coincide to regulate blood flow. We can find classical "negative" and "positive" feedback results:

Negative feedback and pressure control in the cardiovascular control system. The stimulation of the aortic $\left(P_{A}\right)$ and the carotid baroreceptors $\left(P_{C}\right)$ induces a vagal activation, see (19), sympathetic inhibition, bradycardia, diminished cardiac contractive force, vasodilatation on the vessels and a fall in mean blood pressure revealing a multi-input multi-output blood pressure control feedback in the cardiovascular system.

Positive feedback and flow control in the cardiovascular control system. Malliani and co-workers, stretching the thoracic aorta $\left(P_{T}\right)$, produced a significant increase in both heart rate and blood pressure, revealing a positive reflex $[8,12]$. In our point of view, the function of the heart is to pump the blood into the vascular system and the blood flow depends on the pressure difference between ventricular and aortic pressure (usually called the after-load). It is likely that the function of the stretch receptors in the thoracic aorta is to measure the pressure against which the heart has to pump the blood. Then the role of the positive feedback is to control the heart function in order to maintain the blood flow into the vascular system. Therefore, it is obvious that stretching the thoracic aorta can induce reflex changes opposite to those which would be obtained by stretching a carotid sinus [9]. By (19), an increase in $P_{T}$ yields to an increase in $u_{T L L_{R, n+1}}$ trigging off a vasoconstriction in the trunk and lower limbs vascular compartment. In conclusion, assuming that ANS controls the blood flow in the cardiovascular system we are able to explain the apparently contradictory results of these two experiences.

\section{Conclusions and Perspectives}

The decentralised structure of the controller begins to be understood. Cardiovascular modelling leads to the definition of several baroreflex control loops and to a time-discrete approach for the estimation of their sensitivities. So far, only the blood pressure-RR baroreflex control loop has been widely investigated, neglecting the others. In fact, several non invasive signals of the cardiovascular system are nowadays availables: EKG (electrocardiographic signal); peripheral blood pressure (by FINAPRESS for example); photo-plethysmography by which it is possible to estimate local vascular volume; impedance-plethysmography and echocardiography by which it is possible to estimate cardiac output; oronasal thermistor, thoracic and abdominal movement sensors can be used to estimate the respiratory flow and volume. We believe that further model-based analysis of the available signals could allow the proper definition and estimation of other baroreflexes regulating stroke volume, peripheral resistance and compliance. The analysis of these baroreflexes can provide further and complementary information on ANS control of cardiovascular system in many real-life conditions.

\section{REFERENCES}

[1] J. Bestel. Modèle différentiel de la contraction musculaire contrôlée. Application au système cardio-vasculaire. PhD thesis, Université Paris-IX Dauphine. U.F.R. Mathématique de la décision., 2000.

[2] J. Bestel, F. Clément, and M. Sorine. A biomechanical model of muscle contraction. In MICCAI 2001. Fourth International Conference on Medical Image Computing and Computer-Assisted Intervention, 15-17 October 2001. Utrecht.

[3] YC. Fung. Biomechanics. Mechanical properties of living tissues. Springer, second edition, 1993.

[4] AC. Guyton, JM. Ross, O. Carrier, and JR. Walker. Evidence for tissue oxygen demand as the major factor causing autoregulation. Circulation research., Vols XIV and XV (Supp I):I60-I69, 1964.

[5] F.C. Hoppensteadt and C.S. Peskin., editors. The heart and Circulation, chapter 5, pages 105-138. Springer-Verlag in Applied Mathematics, 1992.

[6] E. Koushanpour. Baroreceptor process in the carotid sinus. In Regulation and control in physilogical systems, pages 350-354. Instrument society of america pittsburg edition, 1973.

[7] E. Koushanpour. Baroreceptor discharge behaviour and resetting. In Baroreceptor Reflexes. Integrative Functions and Clinical Aspects, pages 9-44. Springer-verlag edition, 1991. 
[8] A. Malliani. Cardiovascular sympathetic afferent fibers. Rev Physiol. Biochem. Pharmacol., 94:11-74, 1982.

[9] A. Malliani. Principles of cardiovascular Neural Regulation in Health and Disease. Kluwer Academic Publishers, Netherlands, first edition, 2000.

[10] A. Monti and M. Sorine. Baroreceptor modelling and a possible role of the sympathetic efferent activity. In ECMTB 2002. Mathematical Modeling \& Computing in Biology and Medecine, July 2-6 2002. Milan.

[11] JT. Ottesen. Nonlinearity of baroreceptor nerves. In Springer-Verlag, editor, Surveys on Mathematics for Industry, volume 7 (3), pages 187-201. 1997.

[12] M. Pagani, P. Pizzinelli, M. Bergamaschi, and A. Malliani. A positive feedback sympathetic pressor reflex during stretch of the thoracic aorta in conscious dogs. Circ. Res., 50:125-132, 1982.

[13] M. Sermesant, Y. Coudière, H. Delingette, N. Ayache, J. Sainte-Marie, D. Chapelle, F. Clément, and M. Sorine. Towards modelbased estimation of the cardiac electro-mechanical activity from ecg signals and 4d medical images. In MS4CMS'02 "Modelling Es Simulation for Computer-aided Medecine and Surgery". EDP Sciences, SMAI 2002, Rocquencourt, 12-15 november 2002.

[14] N. Stergiopulos, BE. Westerhof, and N. Westerhof. Total arterial inertance as the fourth element of the windkessel model. $A m$ J Physiol., 276 (45):H81-H88, 1999.

[15] N. Stergiopulos, DF. Young, and TR. Rogge. Computer simulation of the arterial flow with applications to arterial and aortic stenoses. j. Biomechanics., 25 (12):1477-1488, 1992.

[16] MF. Taher, ABP. Cecchini, MA. Allen, SR. Gobran, RC. Gorman, BL. Guthrie, KA. Lingenfelter, SY. Rabbany, PM. Rolchigo, J. Melbin, and A. Noordergraaf. baroreceptor responses derived from a fundamental concept. Annal of Biomedical Engineering, 16:429-443, 1988

[17] SM. Toy, J. Melbin, and A. Noordergraaf. Reduced models of arterial systems. IEE Trans on Biomed Eng, BME-32 (2):174-176, 1985. 